\title{
Interactive comment on "Comparing
} Intensity-Duration-Frequency curves derived from CMORPH and radar rainfall estimates over the Eastern Mediterranean" by Francesco Marra et al.

\section{Anonymous Referee \#1}

Received and published: 11 December 2016

\section{Dear Editor,}

Manuscript entitled "Comparing Intensity-Duration-Frequency (IDF) curves derived from CMORPH and radar rainfall estimates over the Eastern Mediterranean" has been reviewed. In the paper, the authors compared IDF curves from radar and CMORPH in different climatic regions. They found that radar shows thicker tail distributions than CMORPH across the Eastern Mediterranean. The authors do not present a new tool. However, the manuscript is well organized. I divided my review into general comments and specific ones. 


\section{General comments:}

1) The Koppen-Geiger classification used are not mentioned or described in section 2.1 .

2) The lack of precision on the applied data leads to misunderstand some results: if the authors are using adjusted CMORPH gauge, what's the reason for analysis the unadjusted CMORPH data (in the same spatiotemporal resolution). I think there is something missing to clarify the methodology.

3) How is spatial (upscaled) data thought to affect precipitation (mainly IDF for return periods)? It is not clear to the readers without background knowledge of the appropriate upscaling method over the study area. Does an adopted upscaling approach perform better than some other techniques (this may not be necessary, but it should be discussed at least)?

4) Any thoughts on the reason why upscaling data is only found significant on the shape parameter (Section 4.1.1)? Maybe I am missing something in this part, but that is my understanding.

5) On the same subject, what is the CMORPH spatial upscaling (downscaling) impacts on precipitation?

6) Why overload figures with features with the insufficient discussion in the text (e.g., Lack of enough discussion for location and scale parameters or for a different time interval in Fig 4)?

The figures axis could be improved if the authors want to make it clear to the reader. Also, I suggest the same size intervals in Y-axis (e.g., Fig 2\&4).

7) Is there any physical link that could impacts on estimations due to different spatial resolution?

8) Some unnecessary materials: in my opinion, it is completely unnecessary to have 
Figure 9, when it is already well-known that the uncertainties increase for longer return period.

9) Finally, I would like to draw your attention to a somewhat old, but still relevant, review on methods to IDF and DDF curves and their uncertainties that might have some valuable lessons in it: Aart Overeem, Adri Buishand and Iwan Holleman, Journal of Hydrology, 2008, 348, pp 124-134

Specific Comments:

Page 5- Line 15:

- I would expect to introduce parameters and explain a little bit how they were computed.

- It is not clear which method used for estimating the GEV distribution parameters by maximum likelihood.

- The bootstrap was applied to assess this uncertainty of record length. This method considers only the uncertainty for the return periods (it's my understanding). What about the uncertainty of the estimation of the GEV parameters (i.e. sampling errors)?

- The assumptions regarding the convenience of the GEV to represent the AMS distribution through the Fisher-Tippett theorem, as well as calculation procedures, would be useful to present in the Appendix.

\section{Page6- Line 12:}

- Figure 2: Estimates of the GEV parameters could be present by Box-Whisker plot that obtained from 999 bootstrap samples, as well for 25th and 75th percentiles of the bootstrap samples. It is also suggested to present the parameter variation against the time by a linear representation that could help the readers to grasp the message from the figures.

Printer-friendly version

- How are different climatic regions thought to affect precipitation (location and scale 
parameters)? It should be discussed at least (regarding figure 2).

Page 6-

- The location and scale parameters are not addressed in details. It is not clear to the reader without background knowledge of the GEV df.

\section{Page 8-}

- I am totally lost. What's the link between section 4.4 with previous sections?

Page 12- Line 13:

- Sorry if I missed this, how is the coverage of upscaled radar and CMORPH pixels? Are they exactly the same? Please, explicitly specify their spatial coverage.

Page 18-

- What's the dashed line in Fig 2 for shape parameter?

Page 20-

- Appendix A. The legend and description in caption do not show similar things (check).

Page 25-

- Why Fig 9 presented? It is well known that confidence in a return level decreases rapidly when the period is more than about two times the length of the original data set. Fig 9 could be removed.

Interactive comment on Hydrol. Earth Syst. Sci. Discuss., doi:10.5194/hess-2016-597, 2016. 